\title{
Una interacción entre la universidad con las comunidades a través de técnicas artesanales en procesamiento de alimentos
}

\author{
An interaction between the university and the communities through artisan \\ techniques in food processing
}

\author{
Lizzi Arelis Martínez González \\ lizziunesr@gmail.com \\ Código ORCID: 0000-0002-8450-1640 \\ Universidad Nacional Experimental Simón Rodríguez, \\ Venezuela
}

\author{
María Adilia Ferreira de Bravo \\ mferreiradebravo@gmail.com \\ Código ORCID: 0000-0002-3219-866X \\ Universidad de Carabobo, Venezuela
}

Recibido: septiembre 2019 / Arbitrado: octubre 2019 / Publicado: enero 2020

RESUMEN

El objetivo de esta investigación fue proponer un plan de capacitación sobre técnicas artesanales en procesamiento de alimentos como interacción de la Universidad Nacional Experimental Simón Rodríguez y la Comunidad de Palmero Municipio Ezequiel Zamora, estado Cojedes- Venezuela. Se fundamentó en la agroecología y la teoría del aprendizaje constructivista de Piaget, con un nivel descriptivo, modalidad Proyecto factible, tomando como población a los habitantes de la Comunidad de Palmero. La técnica empleada fue la encuesta con un cuestionario tipo Likert, el cual permitió diagnosticar la necesidad de un plan de capacitación sobre técnicas artesanales en procesamiento de alimentos, con una confiabilidad Coeficiente Alpha de Cronbach (0.85).Los resultados fueron favorables: la obtención de la interacción universidad con la comunidad, fomentando el consumo de alimentos agroecológicos, exaltando el cuido del medio ambiente, mejorando así la calidad de vida.

Palabras clave: Técnicas artesanales; procesamiento de alimentos; interacción universidad - comunidad

\begin{abstract}
The objective of this research was to propose a Training Plan on Artisanal Techniques in Food Processing as an interaction of the Simón Rodríguez National Experimental University and the Community of Palmero, Ezequiel Zamora Municipality, Cojedes State. It was based on Piaget's agroecology and constructivist learning theory, with a descriptive level, Project Feasible modality, taking the inhabitants of the Palmero Community as population. The technique used was the survey with a Likert-type questionnaire, which made it possible to diagnose the need for a training plan on artisan techniques in food processing, with a reliable alpha coefficient of Cronbach (0.85). Managing to design the training plan yielding favorable results, such as obtaining university interaction with the community, promoting the consumption of agroecological food, exalting care for the environment, thus improving the quality of life.
\end{abstract}

Keywords: Artisan techniques, food processing, university-community interaction 


\section{INTRODUCCIÓN}

Desde el principio de la humanidad, el hombre tiene la necesidad de alimentarse con el fin de satisfacerse y conservar la energía requerida para hacer las actividades de la vida cotidiana, por lo tanto, ha desarrollado estrategias con el paso del tiempo que le han permitido encontrar los alimentos dando un mejor uso a la tierra. Ahora bien, existen países donde las leyes van en beneficio del desarrollo agrícola en el fortalecimiento de la economía, tomando en cuenta la fertilidad de las tierras y el uso adecuado de los espacios. Venezuela ha sido un país privilegiado por la naturaleza, al contar con una geografía con suelos aptos para las diversas actividades económicas. Al respecto, Gómez (citado por Hidalgo, 2010), señala:

Hasta la década de los años 30, el sector agrícola producía todo para atender el consumo nacional como para exportar excedentes considerables de productos de origen vegetal y pecuario, tales como: Café, Cacao, Cuero, Tabacos entre otros...durante el transcurso de la reforma agraria de 1960 ha surgido un fenómeno paradójico de concentración de la actividad privada, pues en realidad al campesino se le ha beneficiado fundamentalmente delas ventas de sus bienhechurías y no de una sustancial dotación de tierra. Esta aseveración permite explicar parcialmente la continuada ruralización del agro, hecho continuo y creciente, lo cual le confiere ya un carácter drástico al sector, cuando se observa que las condiciones de la producción no cuentan ya con campesinos, si no con obreros rurales, cuya relación de familia, ambiente y comunidades han modificado radicalmente. (p. 17)

Es de hacer notar que el país tuvo una baja en la producción de alimentos a partir de la década de los años 30, debido al desplazamiento del trabajo de campo por la actividad privada, trayendo como consecuencia la desruralización del agro, por ende, el abandono del campo. Tal aseveración la hace Delgado (2015):

... pero una vez que se cuenta con vastas
extensiones de tierras incorporadas en
forma harto insatisfactoria, se otorgan al
campesino, (la mayoría con título
precario o con título provisorio), parcelas
de superficies demasiado pequeñas,
cuya posibilidad de explotación
económicamente favorable está lejos de
la realidad. (p.1)

Sin embargo, en la actualidad existe un proceso de transformación social que atraviesa la República Bolivariana de Venezuela, donde el tema de la soberanía alimentaria, el medio rural y muy especialmente los campesinos, como sus principales habitantes, son ineludiblemente convocados a construir un nuevo modelo de desarrollo rural sostenible. Así mismo, dentro de las comunidades se presenta una serie de problemas sociales, educativos, económicos, políticos y culturales; lo cual puede influenciar de manera negativa para alcanzar una mejor calidad de vida de todos sus habitantes, por tal motivo, se deben buscar alternativas factibles en orientación, enseñanza y motivación, con el propósito de ofrecer soluciones a la problemática existente.

Las comunidades rurales necesitan de una comprensión y disposición de solidaridad mutua; partiendo desde la comunidad universitaria se le cuestiona su funcionamiento como también la formación educativa, mientras que a la comunidad rural se le cuestiona la falta de una educación integral e integrada y su poca participación en la búsqueda de mejorar su condición social; siendo factible manifestar mediante un tratamiento que engrane a la educación formal con la informal, se aprovecharía al máximo ambos sectores de la vida nacional, transformando recursos en servicios disponibles, con ejecución de programas, 
proyectos y planes afectos a la colectividad en general.

En este orden de ideas, las Universidades pasan a ser un vehículo comunicante necesario para la transformación, así lo plantea la Ley de Universidades (1970) en su Art. 3:

Las universidades deben realizar una
función rectora en la educación, la
cultura y la ciencia. Para cumplir esa
misión, sus actividades se dirigirán a
crear, asimilar y difundir el saber
mediante la investigación y la
enseñanza; a completar la formación
integral iniciada en los ciclos
educacionales anteriores; y a formar
los equipos profesionales y técnicos
que necesita la nación para su
desarrollo y progreso. (p. 3)

Dentro de este marco, en las universidades existen singulares recursos de vital importancia, entre ellos: humanos, infraestructura física, científicas, culturales, académicos, tecnológicos, pedagógicos e instrumentos y equipos accesibles al habitante de la comunidad rural, a través de la educación informal fuera del aula, basada en la filosofía de extensión. Por consiguiente, se debe incorporar a la sociedad civil de manera organizada impulsando la autogestión de la comunidad rural y aplicar una oferta sistemática de atención, donde se proyecte una mejor realidad rural, atendiendo no sólo el recurso potencial, sino también el factor dinámico en el proceso de cambio, debido a que la complejidad del medio rural no es solo técnica, sino también se manifiesta en términos de comportamiento, actitudes y valores diferentes al ambiente formal.

Sin embargo, el cuestionamiento nacional a la educación universitaria por el no cumplimiento de sus objetivos básicos: docencia, investigación y extensión, su alto costo de financiamiento por parte del Estado; además de su escasa participación en el proceso productivo del medio rural y el incremento de la política partidista en la comunidad universitaria; configuran un comportamiento expresado en falta de credibilidad por parte de la comunidad hacia la universidad.

No existe una verdadera concatenación entre la educación formal y la extensión; a manera de consolidar el proceso educativo entre la educación superior dirigida al área agrícola con la comunidad rural. De este modo, la extensión, se presenta como un elemento importante dentro del proceso educativo dirigido a las comunidades, y es la herramienta para generar la articulación entre la universidad y la comunidad, impulsando la promoción de oportunidades con la organización de la comunidad en procura de una mejor calidad de vida.

Dentro de este proceso, según el Manual de Organización y Funciones Gerenciales de la Universidad Nacional Experimental Simón Rodríguez (1995), la extensión juega un gran papel, por cuanto se establece: "la interacción Universidad - Comunidad - Universidad, en donde se involucran docentes, estudiantes, obreros, curriculum, entre otros, en la promoción de la elevación cultural y social de las comunidades" (p.9). Esto se concreta, interactuando ambas en función de generar una práctica educativa desde la extensión, logrando acciones en donde se promueve, tanto el desarrollo comunitario, como el desarrollo científico - humanístico, en todos los campos del conocimiento en todos los espacios (cultural, económico, social y educativo), involucrando así a todos los actores sociales.

Por consiguiente, la extensión dentro de la UNESR, tiene una función esencial, así como también la docencia y la investigación, ya que permite optimizar la práctica educativa contextualizándola dando respuesta científica y humanística a las necesidades de las comunidades. Por tal motivo, con los programas de extensión se puede obtener propuestas de solución a las necesidades de las comunidades fomentando la 
participación ciudadana, logrando así esa interacción social en pro del desarrollo de los actores del proceso.

Dentro de ese marco, la República Bolivariana de Venezuela es un país poseedor de una gran diversidad de recursos naturales que deben ser aprovechados por las comunidades rurales y así obtener un mejor desarrollo económico de la nación en pro a la solución de problemas requiriendo una mejor participación y liderazgo. Actualmente el Estado Venezolano, desarrolla políticas hacia un modelo de desarrollo rural, sobrestimando los insumos materiales del desarrollo (créditos, maquinarias, infraestructura, insumos agrícolas y subsidios oficiales) y estimula los insumos agrícolas intelectuales (el conocimiento apropiado y apropiable); por ello, las iniciativas del trabajo de extensión se pueden enfocar hacia el apoyo del trabajo agropecuario aportando estrategias de aprendizaje sobre el manejo del área.

Si bien es cierto, el medio rural cuenta con algunas ventajas comparativas y competitivas; una de ellas son las escuelas públicas, ellas actúan en función de vaso comunicante de los conocimientos hacia el desarrollo local, otra son los programas por parte del Estado, en busca del aumento de la producción agropecuaria como sustentabilidad, ejemplo el Programa Todas las Manos a la Siembra (PTMS), cuya finalidad es la producción de alimentos por medio de la agroecología. La conducta actual muestra la población urbana frente al agotamiento de los recursos y ecosistemas naturales, es diferente a la observada en las culturas campesinas donde éstas últimas sienten el problema como una amenaza en el medio donde viven, el campo rural; con todos sus elementos conformados sienten la necesidad de buscar nuevas alternativas de producción que mejoren la calidad de vida, donde el trabajo de extensión puede ser una vía para brindar los conocimientos necesarios, y así proponer soluciones a las problemáticas existentes.

La adquisición de prácticas mejoradas o habilidades, el aumento de conocimiento además del trabajo cooperativo son cambios que se van logrando a través de la influencia del extensionista en la comunidad. Así mismo la diferencia entre el éxito y el fracaso de las iniciativas de desarrollo actual a futuro, necesariamente estará muy ligada a la forma generada, se comparte y se aplica el conocimiento.

El extensionista es una persona consciente de los problemas de la comunidad logrando resultados positivos a través de la organización, debe tener liderazgo, creatividad, deseos de transmitir, compartir, generar conocimientos y motivación.

Dentro de este marco, en la Universidad Nacional Experimental Simón Rodríguez existen recursos similares de vital importancia, entre ellos: humanos, infraestructura física, científicas, culturales, académicas, tecnológicas, pedagógicas e instrumentos y equipos accesibles al habitante de la comunidad rural, por ello al llevar la educación informal fuera del aula asentados en la extensión utilizada como una herramienta de nivel de formación significativo de los estudiantes de las diferentes carreras universitarias, estos basados en el principio del constructivismo, se obtendría que el estudiante construye su propio aprendizaje de acuerdo a su conocimiento.

Cojedes es un estado llanero, con grandes extensiones de tierra que sirven para el trabajo agrícola, existen zonas rurales con carencia de recursos (educación, alimentación, salud, ingresos económicos, etc.), con bajo índice poblacional. La comunidad de Palmero Municipio Ezequiel Zamora del estado Cojedes, cuenta con una población de sesenta (60) habitantes aproximadamente, y posee una tradición histórica importante en el progreso del Estado, primeramente, porque se encuentra el 
Río Tirgua en el límite oeste de la comunidad. La zona presenta sembradíos, una escuela rural, pequeños lotes de terreno, con suelos fértiles y diversos ejemplares de fauna y flora, además de contar con gente cordial, amable y organizada.

La comunidad estudiantil tiene una población de diecinueve (19) estudiantes, concentrada en primera y segunda etapa de educación (de primero a sexto grado). Cabe destacar, la escuela no cuenta con la dotación de servicios necesarios para lograr el desarrollo normal de sus actividades. Así mismo la infraestructura de la institución no se encuentra en las mejores condiciones de seguridad. Ésta comunidad por la distancia a la ciudad, se encuentra afectada por la carencia de los servicios públicos como son: red de cloacas, agua, y empleo de mano de obra asalariada, lo cual no permite el desarrollo integral de la población. La mayoría de la población trabaja con la agricultura desde sus hogares con los llamados conucos, siendo su principal sustento de alimentos.

Por todo lo antes expuesto, se propuso la viabilidad y la alternativa o alternativas necesarias para establecer la integración entre la universidad y la comunidad, por medio del proceso enseñanza y aprendizaje sobre técnicas artesanales en procesamiento de alimentos, en pro del desarrollo del sector rural de la región además de incrementar la sustentabilidad.

En función de ello surgió el objetivo principal para este estudio: Proponer un plan de capacitación sobre técnicas artesanales en procesamiento de alimentos como interacción de los miembros de la universidad con la comunidad; el mismo se derivó de la ejecución de: a) Diagnosticar la necesidad de un plan de capacitación sobre técnicas artesanales en procesamiento de alimentos dirigido a los habitantes de Palmero; b) determinar la factibilidad del estudio y c) diseñar un plan de capacitación sobre técnicas artesanales en procesamiento de alimentos como una interacción entre la universidad con la comunidad.
La universidad tiene una tarea fundamental, formar y capacitar para lograr así el progreso de la nación, obtener el desarrollo del sector agrícola en el Estado Cojedes, no puede ser pensado sin la participación activa de los institutos encargados de formar el recurso en esa área. En este sentido Ferreira (2018) señala, "se hace necesario que el docente de aula del siglo de XXI aplique una didáctica cónsona con los requerimientos de una educación globalizada, inclusiva e integral, que cuestione la raíz de las cosas y enseñe a sus estudiantes hacerlo" (p. 175).

Se exige entonces por parte de la universidad contar con un cuadro de profesionales, personal docente y de investigación de alto nivel, garantizando la excelencia y la pertinencia del mismo, fomentando tanto en los participantes, como en la comunidad rural el espíritu cooperativo a través de actividades de extensión garantizando la consecución de los objetivos extensionistas de la universidad. Dentro de esta perspectiva, lo provechoso de la educación, formal e informal, de la participación y acción comunitaria en la búsqueda del bien común en las zonas rurales, es la ayuda a los pobladores a conocer la problemática en que están inmersos, siendo parte del desarrollo de su población.

Un antecedente relevante para este estudio fue el realizado por Silva y Rincón (2014), en su trabajo titulado "Propuesta de integración para generar proyectos productivos en la comunidad del Barrio Paraíso de la Ciudad de Bogotá D.C.", teniendo como objetivo generar un proyecto productivo auto sostenible como desarrollo de alternativas de práctica social en la comunidad del Barrio Paraíso de la ciudad de Bogotá D.C., llegando a la conclusión que la integración de las universidades con las comunidades permite generar proyectos de alto valor haciendo práctica social colocando a prueba los conocimientos adquiridos en su formación académica complementado por un crecimiento ético y moral. Ésta investigación posee significativa relevancia 
con el presente estudio, porque genera esa integración de las universidades con comunidades más necesitadas para llevar esa educación informal generando proyectos cubriendo las necesidades de la misma.

Existe, pues, un cambio de las relaciones sociales de producción, donde la dignificación de la población mundial realiza un enorme esfuerzo en fortalecer todo aquello que conlleve a la sostenibilidad de la familia dedicada a la agroecología, tanto en los territorios rurales, como indígenas, urbanos y periurbanos a través de procesos que aseguren sus formas de producción y estabilización en sus propios espacios de vida.

Dentro del contexto anteriormente desarrollado, se enmarca la tendencia a realizar proyectos que favorezcan el fomento de actitudes valorativas del ambiente a través de la aplicación de métodos y procedimientos de producir con menores riesgos en la salud y menos impacto en el ambiente, aprovechando al máximo los procesos naturales y los recursos disponibles.

En Venezuela uno de esos métodos se da en el Programa Todas las Manos a la Siembra, cuyo objetivo principal es impulsar la producción agropecuaria, pero de una manera agroecológica, logrando así la sustentabilidad, pero a la vez cuidando el medio ambiente que nos rodea, obteniendo una alimentación saludable, promoviendo cambios en las costumbres alimenticias, incentivando a la siembra desde las escuelas y universidades.

El procesamiento de alimentos se encarga de transformar materias prima: frutas, hortalizas, leche, carnes, granos, semillas, entre otros en productos procesados, llamados productos agroindustriales, pero industrialmente pueden ocasionar daños en la salud de los consumidores, además de ocasionar contaminación por la cantidad de químicos incorporados para obtener mayor número de productos.

Por otra parte, es necesario enfatizar cuando se habla de procesamiento de alimentos a nivel artesanal, se habla de elaborar los productos de una manera orgánica sin químicos contribuyendo a las comunidades en general al mejoramiento en la calidad alimenticia, consumiendo alimentos orgánicos de excelente calidad, y así adquirir esos conocimientos que sirven de apoyo para obtener una mejor calidad de vida de quienes se integren a este proyecto, ya sea por su consumo o también el poder comercializar los excedentes a otros miembros de la comunidad a menores precios organizando pequeñas microempresas.

Dentro de los alimentos procesados artesanalmente existen una gran variedad provenientes tanto de la producción animal como vegetal, transformándose en materia prima para luego ser procesados.

Se trata de propiciar un desarrollo más endógeno, es decir, el desarrollo debe estar basado en el uso racional y en el incremento de la productividad de los recursos que los habitantes realmente disponen, aunque sean escasos; más autogestionario de modo que tengan los conocimientos, habilidades y actitudes necesarios para su elaboración para aportar posibles soluciones.

De todo lo planteado, es importante señalar la necesidad de incorporar procesos alternativos que ayuden a la sustentabilidad de las comunidades, logrando así el desarrollo del país, generando satisfacciones a las necesidades de salud, alimentación y no menos importante, un medioambiente saludable.

\section{MÉTODO}

El nivel o grado de profundidad de la investigación fue de campo, con un enfoque cuantitativo, bajo la modalidad de proyecto factible, definida según el Manual de Trabajos de Grado de Especialización y Maestría y Tesis Doctorales de la Universidad Pedagógica Experimental Libertador (2014): "la elaboración y desarrollo de una propuesta de un modelo 
operativo viable para solucionar problemas, requerimientos o necesidades de organizaciones o grupos sociales" (p.21).

Ahora bien, la investigación se basó en una investigación bajo un nivel descriptivo con un diseño no experimental, la cual se realizó en tres (03) fases: la primera es el diagnóstico, el cual determinó que los habitantes de la comunidad de Palmero tenían la necesidad de formarse en técnicas artesanales en procesamiento de alimentos, esto se realizó a través de la aplicación de un instrumento de recolección de datos, la segunda fase fue la de determinación de la factibilidad que generó la implementación de la investigación y como tercera fase es la formulación de la propuesta para la cual se diseñó un plan de capacitación sobre técnicas artesanales en procesamiento de alimentos como interacción de la universidad y la comunidad.

La población estuvo conformada por 60 habitantes de la comunidad de Palmero Municipio Ezequiel Zamora, estado Cojedes. Una vez definida la misma, se procedió a calcular la muestra utilizando el tipo de muestreo estratificado, para distribuir el tamaño muestral entre los estratos, se tomó en cuenta el criterio afijación no proporcional.

Una vez establecido el diseño de la investigación, se determinó la técnica y el instrumento que permitieron obtener los datos de la realidad; cuya técnica de recolección de datos que se utilizó fue la encuesta y el instrumento utilizado fue el cuestionario, bajo la escala de actitudes tipo Likert; cuya validez se determinó mediante el juicio de experto. En la investigación se comprobó la confiabilidad del instrumento a través del método del Coeficiente Alpha de Cronbach, obteniendo como resultado el valor de 0,85 dando como criterio de decisión para la confiabilidad del instrumento una dimensión muy alta.
RESULTADOS

Se pudo observar que la Comunidad de Palmero Municipio, es netamente rural; donde su principal actividad es la agricultura, es una zona donde los suelos son óptimos para la siembra, y existe gran diversidad de fauna y flora; además de contar con el cauce del Río Tirgua, es el más importante del Estado por ser suministrador de agua de los Estados Carabobo, Aragua y Cojedes.

Tiene como cultura el cuido del medio ambiente, evitando la contaminación para así no afectar a la comunidad, pero por ser una zona turística, en ocasiones existe poca importancia en el cuido del ambiente, trayendo como consecuencias problemas ambientales en la comunidad antes descrita.

Ahora bien, afirmando sobre la base del diagnóstico realizado a los sujetos que conforman la muestra de estudio, se puede señalar que la agricultura es la principal actividad que genera recursos económicos y fuentes de empleo, siempre tienen el suministro de alimentos ya sea de origen animal como de origen vegetal, pero no son alimentos en su totalidad sanos, ya que no utilizan de forma agroecológica los procesos de siembra y de cultivo de los alimentos para el área vegetal, y el área animal, al utilizar alimentos concentrados para abastecer la demanda alimenticia de los animales, por tal motivo no utilizan la agroecología como trabajo de la tierra; es decir que en ocasiones utilizan químicos en el proceso de las actividades.

Un aspecto fundamental es la puesta en práctica de los saberes ancestrales, al fomentar el conocimiento que va de generación en generación, para las actividades de campo, haciendo que exista mayor efectividad en las labores como una tradición familiar, teniendo mayor productividad generando el aprovechamiento de todos los recursos, 
aumentando la autonomía alimentaria en la familia, generando un aporte al desarrollo sustentable de la comunidad.

Con respecto al procesamiento de alimentos, se observó el poco conocimiento en la realización de los productos, teniendo sólo la producción vegetal y animal, más no el proceso agroindustrial, quedando únicamente la materia prima para el consumo y la comercialización. Al determinar la viabilidad de la creación de la propuesta para promover las técnicas artesanales en procesamiento de alimentos en la comunidad de Palmero, se observa que implementaría el consumo de alimentos procesados de una manera sana y segura, aportando a la economía como al desarrollo de la comunidad, debido a que incentivaría la autogestión en el área de producción de alimentos procesados; además por ser una zona turística apoyaría al proceso de desarrollo sustentable, pero sin afectar el medio ambiente, pues son productos libre de químicos agro tóxicos que afectan la salud de los consumidores y la biodiversidad, mejorando la calidad de vida.

Cabe agregar, se implementaría la participación de la comunidad, integrándose con la finalidad de mejorar la comunicación y relación de los habitantes, además de implementar la extensión universitaria por parte de la Universidad Nacional Experimental Simón Rodríguez generando un proceso de enseñanza - aprendizaje por medio de la educación no formal.

Las opiniones de los habitantes de la comunidad de Palmero permiten concluir es evidente llevar a cabo la propuesta de enseñar la realización de alimentos agroindustriales de forma artesanal con la finalidad de promover valores que van en pro del bienestar social, el desarrollo y la sustentabilidad de la comunidad.

Ahora bien, como recomendaciones se tiene el realizar programas de formación en el área de agroecología, en todo el proceso de trabajo de la tierra, con la finalidad de obtener materia prima libre de agro tóxicos. Se deben utilizar más estrategias que aumenten la integración por parte de la comunidad. Se deben realizar convenios con las demás Universidades del Estado, para llevar más proyectos de extensión a la comunidad. Hacer programas de formación en el área administrativa con la finalidad de generar microempresarios, los cuales deben ir de la mano con el desarrollo de la comunidad.

De igual manera se deben llevar programas de formación en el área de sensibilización, crecimiento personal y valores a la comunidad con la finalidad de generar mayor calidad de vida en los habitantes de Palmero. Llevar los talleres de formación en el área de técnicas artesanales en procesamiento de alimento en otras comunidades e instituciones educativas para lograr así aportar con el desarrollo sustentable en el Estado, y aportar a la conciencia agroecológica por medio de una alimentación sana.

\section{CONCLUSIONES}

La propuesta está dirigida a la comunidad requiriendo la participación de representantes de la universidad. El éxito de la misma con el esfuerzo y colaboración de los miembros de ambas comunidades, a quienes se les asignará el alcance del principal objetivo de la propuesta. Se trata, por lo tanto, de sostener simultáneamente dos intencionalidades: la intención de mejorar la calidad de los aprendizajes, y la intención solidaria de ofrecer una respuesta participativa a una necesidad social.

La factibilidad radica en cuanto a que el proyecto está asociado con la efectiva satisfacción de los receptores del mismo; con impactos mensurables en la calidad de vida de la comunidad, con la posibilidad de alcanzar objetivos a mediano y largo plazo, y no sólo de satisfacer necesidades urgentes por única vez. En conclusión, éste tipo de experiencias se caracterizan por promover la integración, el compromiso social y la participación ciudadana, 
así como también de crear conciencia en la disminución del consumo de alimentos con alto contenido de productos químicos que afectan la salud del que lo consume.

Desde el punto de vista económico, el cálculo costo-beneficio pudo determinarse como factible de asumir, ya que la Universidad Nacional Experimental Simón Rodríguez cuenta con la mayoría de los recursos necesarios, lo que permite la practicidad y rapidez de la puesta en práctica de cada una de las actividades. De la misma manera el recurso humano necesario para cada una de las actividades está capacitado con la finalidad de optimizar el proceso. La investigación constituye un apoyo que brinda la posibilidad de desarrollo tanto en el plano económico como en el de proyección institucional. Existe gran importancia en lo referente de dar a conocer el trabajo que puede hacer la Universidad Nacional Experimental Simón Rodríguez académicamente al interactuar con las diferentes comunidades aledañas, para lograr así impartir un proceso de enseñanza aprendizaje en pro del desarrollo de la misma.

El propósito de esta propuesta fue diseñar un plan de capacitación sobre técnicas artesanales en procesamiento de alimentos como interacción de la Universidad Nacional Experimental Simón Rodríguez y la Comunidad de Palmero Municipio Ezequiel Zamora, con la finalidad de enseñar a elaborar productos agroindustriales, pero de forma artesanal. Los alimentos procesados aportarían un beneficio para el consumidor, ya que son sanos; además de contribuir con la mejora del medio ambiente y fortalece la economía del hogar, pues sirve de autogestión para el desarrollo sustentable de la comunidad.

La formación contribuye el camino a través del cual se puede integrar la formación de conocimientos, habilidades y valores, que permitan a los facilitadores y miembros de la comunidad, desarrollarse como personas críticas, capaces de participar activamente en la construcción de la sociedad sobre la base de principios de solidaridad, bien común, valoración de la identidad y la integración.

Desde ésta perspectiva, el procesamiento de alimentos se hace cargo de uno de los mayores desafíos, ya que así se va a promover la interacción con los habitantes de la comunidad, para hacer más efectivas las actividades a cumplir, logrando que los habitantes tengan la capacidad para proponer diversas estrategias en la resolución de situaciones problemáticas, generando procesos productivos de forma eficaz y eficiente. Estas acciones deben desarrollarse a través de talleres, para lograr así desarrollar habilidades, destrezas y al mismo tiempo, propicie el pensamiento crítico, reflexivo, humanista y ambientalista, además de formarlo en el campo científico, tecnológico y social, para su incorporación al campo productivo. Así mismo, el proyecto pretende asumir el papel de transformación, desarrollando espacios para las innovaciones tecnológicas, el quehacer comunitario, para la salud y la vida, para la producción, la productividad y la creatividad.

El trabajo comunitario constituye una forma de participación social, una manera de acercarse a los problemas y una actitud de responsabilidad frente a estructuras sociales ya existentes, fundamentándose en el aprendizaje-servicio. Este tipo de experiencia educativa, cuando involucra a otros, más allá de los participantes y los facilitadores, tiene consecuencias sobre el desarrollo personal, afectivo y social, además del académico.

En este sentido, el proyecto permitirá producir productos procesados cárnicos, lácteos, de frutas y de hortalizas, para el consumo, así como para la comercialización, además de poder crear pequeños negocios o microempresas, generando recursos, mejorando el ambiente y condiciones de trabajo, aumentando la satisfacción de los participantes.

Se seleccionó la Comunidad de Palmero Municipio Ezequiel Zamora del estado Cojedes, por estar en las adyacencias del Río Tirgua, una cuenca 
que surte de agua a los estados Carabobo, Aragua y Cojedes; por tal motivo se deben generar estrategias que sirvan de beneficio del cuido del mismo.

Para la realización del plan de capacitación en técnicas artesanales en procesamiento de alimentos, dirigido a la comunidad de Palmero del Municipio Ezequiel Zamora del Estado Cojedes se establecieron unas estrategias para el desarrollo de la misma divido en cuatro (04) fases:

Fase I: Formulación de estrategias basadas en la capacitación sobre técnicas artesanales en procesamiento de alimentos, motivación a la comunidad. Ésta fase corresponde a la introducción donde todos se conocen, y se explica el proceso de todos los talleres que se van a realizar, dando a conocer la Universidad Nacional Experimental Simón Rodríguez y realizando dinámicas de inicio para conocerse unos con otros.

Fase II: Integración de la comunidad en la participación de las diversas actividades relacionadas con la propuesta, con la finalidad de motivar a la comunidad para la realización de los talleres, explicando el beneficio de formarse en el área, además de trabajar el crecimiento personal que es muy importante para la efectividad del proceso.

Fase III: Implementación del programa de capacitación sobre técnicas artesanales en procesamiento de alimentos a través de la ejecución de talleres, que permitan la interacción con la comunidad, dando a conocer los diferentes tipos de procesamiento de alimentos tanto en el área industrial como en el área artesanal, además de la importancia que éstos tienen para el cuido del medioambiente.

Fase IV: Evaluación del plan de capacitación sobre técnicas artesanales en procesamiento de alimentos, a través de los testimonios por parte de los habitantes por medio de lluvia de ideas enfocándose en el área de salud del consumidor, el abastecimiento de alimentos, el cuido del medio ambiente y la integración de la universidad con la comunidad, así como también el fortalecimiento del desarrollo sustentable que se genera con la formación teórico - práctica de los temas a desarrollar.

\section{REFERENCIAS}

Delgado, B. (2015). Consideraciones sobre la Reforma agraria venezolana. Caracas. Recuperado de http://servicio.bc.uc.edu.ve/derecho/revista/ idc19/19-12.pdf

Ferreira de Bravo, M. (2018). Constructo didáctico de la geometría. Una visión de cambio y transformación en la capacidad de abstracción y el pensamiento crítico. (Disertación Doctoral). Recuperado de www.bc.uc.edu.ve

Hidalgo, Y. (2010). Análisis de las políticas agrícolas y sus efectos económicos para el periodo 2008 - 2009 en la parroquia Virgen del Valle Municipio Tucupita del Estado Delta Amacuro. (Trabajo de grado no publicado) Venezuela. Recuperado de https://es.scribd.com/doc/48653282/Tesisde-Politicas-Agricolas-Unefa-Delta-Amacuro

Ley de Universidades. 1970. Gaceta Oficial Nº 1429 (Extraordinario). Caracas, Venezuela. Recuperado de http://www.ucv.ve/fileadmin/user _upload/comision _electoral/ Normativa_legal/index.htm

Silva, D. y Rincón, O. (2014). Propuesta de Integración para generar proyectos productivos en la comunidad del Barrio Paraíso de la Ciudad de Bogotá D.C. Trabajo de Grado de Maestría. Universidad Católica de Colombia

Universidad Nacional Experimental Simón Rodríguez. (1995). Manual de Organización y Funciones Generales. Caracas, Venezuela: Epísteme

Universidad Pedagógica Experimental Libertador. (2011). Manual de Trabajo de Grado de Maestría y Tesis Doctorales. Venezuela 